\title{
2-3 mai à Berne: le rôle de la géographie dans la société ou une géographie corporatiste et acritique.
}

Les 2 et 3 mai a eu lieu à Berne une rencontre nationale organisée sous les auspices de la Commission Suisse de Géographie, de la Société Suisse de Géographie, de la Société Suisse de Géographie Appliquée et de l'Association Suisse des Professeurs de Géographie, regroupant entre 150 et 200 participants. Le thème de ces journées était: Le rôle de la géographie dans la société. Les facettes d'une identité. Afin d'améliorer la piètre image générale de notre discipline et le peu de fonds pour la recherche qui lui sont alloués, le but déclaré était de tendre vers l'établissement d'une "image directrice" de la géographie suisse. Dans le dernier numéro de GEOGRAPHICA HELVETICA (no 2/1986, pp. 97 à 102), nous sont rapportés les termes, recommandations et propositions de cette rencontre.

Nous n'allons pas retracer en quelques lignes ce colloque. Nous voulons simplement traiter trois questions qui nous semblent fondamentales et qui ont été au centre de tous les débats. Il s'agit d'une part de la géographie dite appliquée, d'autre part du corporatisme et enfin de l'autonomie intellectuelle et du rôle critique des sciences de l'homme.

\section{Géographie théorique versus géographie pratique?}

Nous n'entrerons pas en matière sur la conception réductrice de notre discipline que représente la recherche d'une "image" au singulier et de surcroit "directrice".

Passons également sur le fait que l'amélioration d'une "image" nécessiterait avant tout l'analyse du pourquoi de son état actuel. Cependant sur cette question aucun texte et aucun débat n'ont été prévus.

Mais attardons-nous sur une idée générale qui nous parait dangereuse. Elle est synthétisée par les termes "géographie appliquée", "géographie pratique", "géographie opératoire", utilisés in- lassablement dans les textes et les discours de ces deux journées. C'est une conception antinomique et simpliste du rapport entre la théorie et la pratique. Elle véhicule une vision privilégiant le savoir opératoire, excluant de façon implicite la dimension spéculative de la recherche.

La connaissance appliquée versus la connaissance théorique ? La pratique versus l'utopie? Poser le problème en ces termes signifie la remise en cause des recherches qui ne sont pas rentables, indépendamment du point de vue adopté pour définir la rentabilité. Mais de toute évidence ce sont ceux qui ont le pouvoir de décision, les commanditaires, qui décident du sens du mot rentabilité et de sa portée. Quel que soit le régime politique en place, quelles que soient les institutions et les idéologies impliquées, cette conception opératoire du savoir conduit nécessairement à l'étouffement des alternatives au niveau intellectuel.

Le concept de praxis, au sens philosophique, qui lie indissociablement la connaissance et la pratique, nous parait bien plus riche car non exclusif. Un discours, une bribe quelconque de théorie, est alors considéré comme une action sur d'autres discours qui, euxmêmes, ne sont que des visions partielles ou globales du monde. En ce sens un discours est une action sur un système de relations sociales (1). Inversément, une pratique, aussi aliénée soit-elle, présuppose nécessairement une théorie. Cette dernière, en tant que vision du monde, peut être plus ou moins implicite. Disons qu'elle varie socialement et individuellement sur un continuum allant de l'"en soi" (du donné, de l'implicite) au "pour soi" (au conscientisé, à l'explicite). Mais dans tous les cas, sans ce substrat cognitif

Dario Lopreno, Assistant/ Géographie, Université de Genève 
englobant il n'y a pas de possibles ni de représentations du réel.

Cette conception liée à la praxis a un immense avantage. Elle reconnaît, à travers la diversité du social, la différence et la variation des conditions sociales et donc de leurs expressions intellectuelles. Elle laisse la porte ouverte à toute école, tendance, idéologie, à toute approche aussi hérétique ou éloignée de la réalité soit-elle. Elle empêche d'exercer la censure qu'induit un savoir qui se taxe d'"appliqué", victime de la césure aliénante qu'il opère entre la théorie et la pratique.

\section{L'illusion corporatiste}

Un corporatisme classique et explicite a dominé ces deux journées et les documents préparatoires. Dès le départ (2), l'élaboration de cette image directrice reposait entre autres sur "le besoin et l'utilité d'un lien associatif de défense de la discipline" et la nécessité d'un "lobby de la géographie".

On en est venu ainsi à parler de "la géographie" non point pour qualifier une approche donnée des sciences de l'homme, mais pour nommer tous les géographes de Suisse. Ainsi le glissement le plus prévisible s'est opéré avec une aisance et une rapidité tout de même déconcertantes: on s'est mis à évoquer la nécessité de "tenir tête à la concurrance d'autres groupes professionels" (3). Même en se situant il y a sept siècles en arrière, ce type de corporatisme serait dénué de sens. En effet la géographie, affublée ou non de l'étiquette 'appliquée', ne saurait offrir un substrat cohérent permettant d'édifier un corporatisme quelconque sur sa base.

En outre, une question fondamentale a été évacuée d'office. Les géographes ont-ils un intérêt commun ? Celui-ci transcende-t-il les diverses positions sociales possibles et les antagonismes implicites ou explicites qui les traversent? A l'occasion de l'un des discours de conclusion, un orateur nous a même comparés, dans un dérapage conceptuellement incontrôlé, à des paysans devant, par nécessité, créer un lobby pour défendre leurs intérêts économiques et politiques. Le mot corporatisme était là, étalé de long en large... mais ses adeptes n'osaient le prononcer.

Enonçons un exemple banal mais combien significatif. Prenons un géographe, partisan du programme de nucléarisation de la Suisse mis en place par la classe dominante helvétique et le Conseil Fédé- ral, réalisé en faisant appel à la panoplie d'arguments nationalistes, sans reculer devant le recours a la force policière. Prenons également un géographe partisan des mouvements antinucléaires et de leurs moyens de résistance. Y a-t-il vraiment un intérêt économique, politique, ou plus largement intellectuel commun entre ces deux personnes ? Ont-ils une conception unique de la discipline à défendre ? Entre un chercheur qui, nolens volens, conçoit sa discipline comme une justification à apporter à la politique gouvernementale et un chercheur qui la conçoit comme une des armes privilégiées de la critique sociale, y a-t-il un intérêt commun ? Il y en a indubitablement un, commun à tous les géographes et plus généralement à tous les scientifiques, que ces derniers se situent dans les sciences de l'homme ou de la nature. C'est d'obtenir et de sauvegarder jalousement les conditions permettant un échange et un débat incessants, le plus large possible, le plus respectueux des différences sans restriction aucune. Pour cela il est indispensable de reconnastre la diversité des conditions sociales et personnelles. Car c'est d'elle que nait la diversité scientifique. En dehors de ce ciment commun, source de vie de l'imagination dans la science et dans la recherche, il ne saurait $y$ avoir de richesse ni d'honnêteté intellectuelles. Nous pensons que c'est là le seul et unique intérêt commun existant a priori entre deux intellectuels. Ne pas le reconnaitre traduit indubitablement une volonté de censure.

Le corporatisme scientifique est un concept qui implique l'abstraction du contexte social, historique et politique. C'est une vision de la science qui transforme l'organe à raisonner qu'est le cerveau en caisse de résonance du pouvoir politique en place. Cela signifie, ni plus ni moins, la mort de la géographie en tant que science de l'homme.

Sous le corporatisme, le totalitarisme du consensus

Derrière ce corporatisme se profile une grave remise en cause de l'autonomie scientifique et de l'existence de la géographie en tant que critique sociale. La aussi le vocabulaire employé lors de ce colloque a l'avantage d'être tragiquement clair: "harmonisation des travaux", "recherche d'un dénominateur commun", mise au point d'une conception générale pour une "image directrice 'Géo- 
graphie Suisse' "(4). En d'autres termes il s'agit de l'extension de la conception corporatiste au niveau des contenus de la discipline.

On est en droit de se demander dans quel but les organisateurs de ces journées de réflexions et de débats (durant lesquelles réflexion et débat étaient fort mal accueillis) veulent uniformiser la géographie en Suisse. La réponse fut donnée mille et une fois, par oral et par écrit, tel un leitmotiv lancinant. "Les questions relatives à l'environnement ont tendance à occuper toujours d'avantage les détenteurs du pouvoir. Il est donc essentiel que la géographie expose quelle est sa place et sa responsabilité en ce domaine, se fasse connaitre et se préoccupe de démontrer sa contribution" (5). Autrement dit lorsque le pouvoir, ici politique, ailleurs économique, se pose une question, elle devrait être admise d'emblée par les géographes comme étant pertinente, comme méritant d'être posée. Dans ce cas ce serait le pouvoir politique dominant qui nous dicterait nos problématiques...

Mais, peut-on objecter à ce qui précède, l'environnement est un problème réel et le pouvoir ayant, justement, le pouvoir, on ne peut s'amuser à l'ignorer. A cela nous répondons d'une part que la seule garantie de l'autonomie intellectuelle est de toujours se questionner préliminairement sur ia pertinence d'un problème posé, pertinence culturelle au sens large, nécessairement relative à celui qui soulève le problème. Nous pensons d'autre part que cette façon d'aborder la question du pouvoir dans les sciences de l'homme, en partant de son existence en tant qu'Etat, sans prendre en considération les autres sources de pouvoir, non nécessairement dominantes, relève quasi immanquablement d'une soumission plus générale à ce même pouvoir étatique.

Nous en voulons pour preuve les documents préparatoires du colloque. Dans un texte intitulé "Profession: géographe", il est recommandé au géographe de "soigner ses relations militaires également en dehors du service (6) (sic...). Impossible de ne pas penser à l'ouvrage de Y. Lacoste, "La géographie, ça sert, d'abord, à faire la guerre." Impossible de ne pas penser également que le Département Militaire Fédéral est plus riche que Crésus. C'est pourquoi certains géographe qui ont oublié jusqu'à l'existence même du mot ethique ronronnent aux pieds du Moloch fédéral en espérant recevoir quelques miettes de récompense.
Dans un autre texte on regrette que les géographes ne soient pas "consultés sur les politiques à mettre en oeuvre" par les instances politiques fédérales (7). Le géographe, s'il est ici considéré comme politiquement actif, n'est conçu comme actif que dans le cadre des instances gouvernementales. Et, qui plus est, on le pose d'emblée comme consultant, autrement dit comme main-d'oeuvre intellectuelle du pouvoir. Ce n'est pas un hasard si la "géographie opératoire" et la "géographie appliquée" étaient portées au pinacle tout au long des deux journées.

Les géographes ont également été mis en garde: "Si la géographie reste trop éloignée du contexte de la politique scientifique (...) ne risque-t-elle pas de faire les frais des inévitables restrictions budgétaires ?" (8) Connaissant la politique du Fonds $\mathrm{Na}-$ tional de la Recherche Scientifique, rentable, opératoire, efficiente, et inébranlablement fermée à tout projet de recherche contenant ne serait-ce que l'ombre d'une critique quelle qu'elle soit, la question posée ci-dessus prend son sens.

Soumettez-vous, aplatissez-vous, soyez sages, sinon ... plus d'argent. Il y a même eu des interventions préconisant de présenter des projets de recherches, destinés au Fonds National, qui soient politiquement admissibles et assimilables par celui-ci. Cela ressemble redoutablement à une variation sur le thème connu de la "démocratie qui administre, dans la démocratie privée de discussion", cette forme d'anesthésie sociale tant redoutée par l'écrivain Peter Bichsel (9).

Mais on a omis de nous dire une chose. C'est qu'avec cette pratique inféodée "la géographie" s'est fait éliminer des programmes du Fonds National de la Recherche Scientifique. C'est que les géographes ne sont plus représentés nulle part dans les instances fédérales scolaires et de la recherche. Il n'y a pire serviteur que celui qui, par mimétisme, est à l'image de son mâ̂tre. Il n'apporte plus rien au mâtre, puisqu' il le répète et le singe. Il prend beaucoup plus de coups de bâton que tous les autres serviteurs, car le martre ne déteste rien autant que de voir tous ses défauts dans un miroir. Il est celui dont on peut se défaire le plus facilement puisque, étant soumis, il ne causera point de difficultés. 
La géographie en Suisse veut-elle se suicider ?

Le premier jour un orateur a situé la géographie entre projet social et utopie. Le verdict est tombé le jour suivant: l'utopie est bonne pour le long terme, le rêve est un luxe que nous ne saurions nous payer, a-t-il été répondu. Si Candide était passé par là, ne nous aurait-il pas demandé si les diverses "Conceptions Globales" des transports, de l'énergie, de l'armée, etc., du gouvernement fédéral ne sont pas des utopies?

Plus inquiétant que tout ce qui précède fut le vote des "recommandations" finales par l'assemblée des participants (10):

- "Le respect du principe de l'autonomie scientifique a été balayé à une écrasante majorité dans le paragraphe traitant du renforcement "de la position de la discipline dans les cercles de la politique de la recherche".

- "La nécessité de notre apport critique" a été balayée à une écrasante majorité dans le paragraphe traitant de notre "intégration" au Fonds National de la Recherche Scientifique et de "l'état actuel de l'environnement, de la société et de la science."

- En fin de compte fort peu de voix se sont élevées contre cette préparation du suicide de la géographie en tant que science sociale.

L'amnésie semble décidément être le mal de cette fin de siècle. Pourtant l'exemple de Galilée pourrait être édifiant pour les géographes. Affamé, il a continué ses recherches. Même face à la terreur d'être brâlé vif, à cause de laquelle il a abjuré ses recherches, il a affirmé sa conviction en prononçant le merveilleux "et pourtant elle tourne". A la place d'être menacés du bucher, les géographes de notre colloque ne sont qu'indirectement menacés de ne pas recevoir des subventions qu'ils n'ont encore jamais reçues. Et pour cela ils ont cédé.

Revenons à Galilée. L'originalité et la force de sa théorie ont fait qu'au-delà de tous les anathèmes prononcés durant son procès, l'Eglise a entretenu Galilée jusqu'à la fin de sa vie dans une captivité dans laquelle il pouvait (ou devait) continuer ses recherches hérétiques. L'autonomie et la critique intellectuelles semblent décidément plus fortes que la servilité.
N'oublions pas enfin que cette rencontre était destinée à améliorer la piètre image de la géographie en Suisse. Cette fois on pourra affirmer que les géographes ont l'image qu'ils méritent. "Là haut, dans les villas, personne ne pensait à une chose: le vieux monde n'avait pas été écrasé par ses adversaires, il s'était tué lui-même", écrit C. Pavese dans La Casa in collina (11) ...

\section{Pour mémoire}

Avant de conclure, nous tenons à signaler deux erreurs importantes, sinon inquiétantes, qui figurent dans le rapport de la rencontre des 2 et 3 mai à Berne, paru dans le dernier numéro de GEOGRAPHICA HELVETICA.

Premièrement, le texte français met explicitement en avant "l'autonomie scientifique" "comme condition sine qua non" pour notre insertion dans les cercles de la politique de la recherche (12). Est-ce vraiment à nous de rappeler que la mention de l'autonomie scientifique a, malheureusement, été refusée, à une écrasante majorité, le 3 mai, lors du vote du texte publié dans le dernier GEOGRAPHICA HELVETICA ?

Deuxièmement, les deux textes, allemands et français, mentionnent plus loin "la nécessité d'un apport critique" de la géographie face à la politique de la recherche (13). Est-ce vraiment à nous de rappeler que ceci a, malheureusement, également été balayé lors du vote, à une non moins écrasante majorité ?

Notes:

(1) L. J. Prieto: Pertinence et pratique. Ed. Minuit, Paris, 1975, pp 150 et ss.

(2) Dépliant d'invitation et de présentation de la Journée des Géographes, Commission Suisse de Géographie, Société Suisse de Géographie Appliquée, Association Suisse des Professeurs de Géographie, 4 pages.

(3) "Profession: géographe" in "Documentation pour un projet d'une image directrice: Le rôle de la géographie dans la société/ Les facettes d'une identité", documents pour la Journée des Géographes, Berne, avril 1986, p. B-4.

(4) "Introduction" in "Documentation pour un projet...", p.3. 
(5) cf note (2)

(6) "Profession: géographe" in "Documentation pour un projet...", p. B-9

(7) "La géographie dans le contexte de la politique scientifique" in "Documentation pour un projet...", p. D-1

(8) idem p. D-4

(9) P. Bichsel, "La Suisse du Suisse", Ed. L'âge d'Homme, Lausanne, 1970, p. 54
(10) "Propositions pour la poursuite de l'action" recommandations votées en conclusion de la Journée des Géographes des 2 et 3 mai 1986, document distribué au colloque, 4 pages

(11) C. Pavese, "La casa in collina", Ed. Einaudi, Torino, 1967, p. 77

(12) "Le rôle de la géographie dans la société" in GEOGRAPHICA HELVETICA no 2-1986, p. 99, paragraphe $D_{\text {. }}$ alinéa $I$

(13) idem, p. 100

\section{Literaturbesprechung}

HÖFLE Konrad: Bildungsgeographie und Raumgliederung. Das Beispiel Tirol. Innsbrucker Geographische Studien, Band 10, Selbstverlag des Institutes für Geographie der Universität Innsbruck, $152 \mathrm{~S} ., 40 \mathrm{Tab} ., 4 \mathrm{Abb} ., 11$ Karten, 1984, öS 240.--.

Nach den 1980 von Peter Meusburger verfassten "Beiträgen zur Geographie des Bildungs- und Qualifikationswesens" (Innsbrucker Geogr. Studien, Band 7) befasst sich auch der vorliegende Band mit bildungsgeographischen Fragestellungen. Die Basis dieses Druckwerkes bildet die 1982 vom Verfasser eingereichte Dissertation.

Hauptziel der vorliegenden Untersuchung ist es, für das natur- und kulturgeographisch recht differenzierte Bundesland Tirol die räumlichen Strukturen und Prozesse der Daseinsfunktion "SichBilden" in ihren Wechselwirkungen mit dem übrigen geographischen Faktorenkomplex (z.B. Höhenlage, Zentralität, Wirtschafts- und Sozialstruktur) zu erfassen sowie in Text und Karte verständlich zu machen. Zur Anwendung gelangen vor allem statistisch-kombinatorische und kartographisch-synoptische Verfahren. Die Daten beruhen einerseits auf einer gross angelegten, flächendeckenden Fragebogenerhebung, andererseits auf zahlreichen, elektronisch gespeicherten Strukturdaten der Tiroler Gemeinden. Diese Kombination primär- und sekundärstatistischer Daten erlaubte dem Autor die Berechnung aussagekräftiger Ergebnisse.

Die Resultate entsprechen in manchen Punkten auch denjenigen bildungsgeographischen Untersuchungen, die in jüngster Zeit in der Schweiz durchgeführt wurden. So stellt HOFLE beispielsweise fest, dass die wirtschaftlichen Problemregionen Tirols durch die $\mathrm{Ab}-$ wanderung aufstiegswilliger Bevölkerungsschichten dauernd einen Teil der aus ihrem Gebiet hervorgegangenen Absolventen weiterführender Schulen an die zentralen Gunsträume verlieren. Selbst bei Anheben der Bildungsbeteiligung in diesen Passivzonen wird der Anteil der Wohnbevölkerung bzw. der Berufstätigen mit höherer Schulbildung stets zurückbleiben, solange nicht die Erwerbsstruktur in der Heimatregion verbessert wird. Weiter betont HOFLE die Notwendigkeit zusätzlicher beruflicher Weiterbildungsmöglichkeiten in den Problemgebieten im Rahmen einer längerfristigen Bildungsplanung und in Verbindung mit entsprechenden Massnahmen zur regionalen Arbeitsmarktförderung.

GH 3/86 Elisabeth Bühler-Conrad, Zürich 